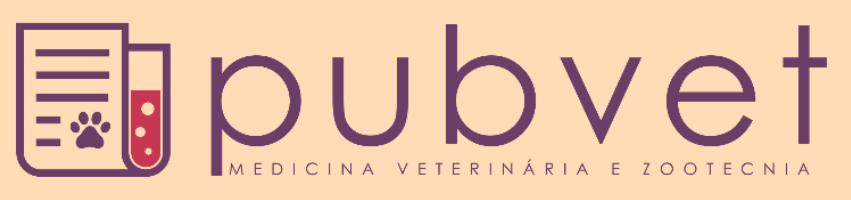

https://doi.org/10.31533/pubvet.v13n3a277.1-10

\title{
Palma forrageira na alimentação de ruminantes
}

\author{
Levi Auto Lopes $^{*}{ }^{*}$, Daniel Barros $\operatorname{Cardoso}^{1 \bullet}$, Karine Silva Camargo ${ }^{1 \bullet}$, Tomás \\ Guilherme Pereira da Silva ${ }^{1}{ }^{\circ}$, Julyana de Sena Rodrigues Souza ${ }^{1}$, José Ricardo \\ Coelho da Silva $^{1}{ }^{\circ}$, Jasiel Santos de Morais $^{1 \bullet}$, Thalita Polyana Monteiro Araújo ${ }^{1 \bullet}$
}

${ }^{1}$ Universidade Federal Rural de Pernambuco, Departamento de Zootecnia. Recife-PE, Brasil.

*Autor para correspondência, E-mail: levi_auto@hotmail.com

\begin{abstract}
Resumo. As diferentes espécies e variedades de palma forrageira configuram recurso alimentar utilizado nas diversas regiões áridas e semiáridas do mundo. $\mathrm{O}$ valor nutritivo dessa planta forrageira oscila em função de uma série de fatores adaptativos atribuídos a aridez, podendo-se destacar as diferenças fenotípicas entre as espécies, variedades ou clones da planta, adubação e tratos culturais, idade, cladódios e tempo de armazenamento após o corte, entre outros aspectos, que juntos terão diferentes implicações na nutrição animal. A palma forrageira apresenta elevada aceitabilidade por ruminantes e grandes quantidades podem ser voluntariamente consumidas, assim como todas as espécies de palma apresentam alta digestibilidade da matéria seca, no entanto devido a diversos fatores que levam a diferenças na composição química e anatômica, pode existir variação quanto à ingestão da ração, digestibilidade e aproveitamento de seus nutrientes. Assim, objetivouse com este trabalho abordar o uso de genótipos de palma na alimentação de animais ruminantes.
\end{abstract}

Palavras chave: cactáceas, consumo, digestibilidade, forragem, semiárido

\section{Spineless cactus for ruminant feed}

Abstract. The different species and varieties of spineless cactus constitute a food resource used in the several arid and semi-arid regions of the world. The nutritive value of this forage plant oscillates due to a series of adaptive factors attributed to aridity, and phenotypic differences between the species, varieties or clones of the plant, fertilization and cropping practices, age, cladodes and time of storage can be highlighted. cutting, among other aspects, that together will have different implications in animal nutrition. Spineless cactus has a high acceptability for ruminants and large quantities can be voluntarily consumed, as all species of palm have high digestibility of dry matter, however due to several factors that lead to differences in chemical and anatomical composition, there may be variation to feed intake, digestibility and utilization of its nutrients. Thus, the objective of this work was to discuss the use of spineless cactus genotypes in the feeding of ruminant animals.

Keywords: cactaceae, fed, digestibility, forage, semi-arid

\section{Nopal en la alimentación de rumiantes}

Resumen. Las diferentes especies y variedades de nopal configuran recurso alimentario utilizado en las diversas regiones áridas y semiáridas del mundo. El valor nutritivo de esta planta forrajera oscila en función de una serie de factores adaptativos atribuidos a la aridez, pudiéndose destacar las diferencias fenotípicas entre las especies, variedades o clones de la planta, fertilización y manejos cultivares, edad, brotes y tiempo de almacenamiento después del corte, entre otros aspectos, que juntos tendrán diferentes implicaciones en la 
nutrición animal. El nopal presenta una elevada aceptación por rumiantes y grandes cantidades pueden ser voluntariamente consumidas, así como todas las especies de palma presentan alta digestibilidad de la materia seca, sin embargo, debido a diversos factores que llevan a diferencias en la composición química y anatómica, puede existir variación a la ingestión de la ración, digestibilidad y aprovechamiento de sus nutrientes. Así, se objetivó con este trabajo abordar el uso de genotipos de nopal en la alimentación de animales rumiantes.

Palabras clave: cactácea, consumo, digestibilidad, forraje, semiárido

\section{Introdução}

Em condições limitantes, como em zonas áridas e semiáridas, um fator que merece destaque é a flutuação produtiva na pecuária de ruminantes em razão da escassez de forragens (Ben Salem \& Smith, 2008). Diante da atual discussão sobre o aumento da temperatura global e contínuo declínio dos recursos hídricos do planeta, presume-se que a palma forrageira se revele com grandes expectativas futuras para alimentação animal e também humano. Este alimento já se destaca como importante recurso forrageiro cultivado, em diversas partes do globo terrestre, devido a aspectos fisiológicos como tolerância e resistência à seca, metabolismo fotossintético MAC e eficiência no uso da água (Silva et al., 2014) e atributos nutricionais, como teor de umidade e conteúdo energético (Batista et al., 2003b; Costa et al., 2012).

O valor nutritivo da palma forrageira pode ser influenciado por uma gama de fatores adaptativos atribuídos a aridez. Segundo Batista et al. (2009), essas adaptações são capazes de modificar suas características químicas e podem ter diferentes implicações sobre o consumo de ração e a utilização dos diferentes nutrientes. Além dos fatores mencionados, podem-se destacar as diferenças fenotípicas entre as espécies, variedades ou clones da planta, adubação e tratos culturais, idade, cladódios e tempo de armazenamento após o corte, entre outros aspectos, que juntos terão diferentes implicações na nutrição animal. Diante do exposto, objetivou-se com este trabalho revisar a utilização de diferentes genótipos de palma forrageira na alimentação de ruminantes.

\section{Palma forrageira}

A palma forrageira pertence à família Cactaceae possui cerca de 130 gêneros e 1.500 espécies (Santos et al., 2013). A utilização de espécies do grupo Opuntia e Nopalea nos sistemas produtivos consiste em uma importante alternativa para alimentação de ruminantes, pois suas características anatômicas e fisiológicas permitiram adaptação às condições edafoclimáticas nas regiões áridas e semiáridas. Atualmente, o Brasil é o país com maior cultivo da palma forrageira do mundo, com área estimada em 600 mil ha (Dubeux Júnior et al., 2013). A palma apresenta grande diversidade genética, apesar de possuir o mesmo tipo fotossintético, os clones de cactos diferem em suas características morfológicas por possuírem diferentes tamanhos e estruturas de cladódios (Barbosa et al., 2018). A literatura cientifica na última década de avaliação de palma forrageira na alimentação de ruminantes destaca, sobretudo, três variedades: cv. Gigante, cv. Miúda e mais recente o cv. Orelha de Elefante Mexicana.

Gigante, Redonda e Clone IPA-20, [Gênero: Opuntia sp., (espécie: Opuntia fícus indica-Mill]. Estes cultivares se destacam pela rusticidade, tolerância à secas intensas e a infestações da cochonilha de escamas Diaspis echinocacti (Santos et al., 1997). A palma Gigante apresenta raquetes ovaladas de cor verde escura com 19 a $28 \mathrm{~mm}$ de espessura, 30 a $60 \mathrm{~cm}$ de comprimento, 20 a $40 \mathrm{~cm}$ de largura, pesando entorno de $1,8 \mathrm{~kg}$ e recobertas por uma camada de cera. Estas plantas têm porte arborescente com 3 a 5 $\mathrm{m}$ de altura (Marques et al., 2017). Entretanto, a ocorrência da cochonilha do carmim tem promovido acentuada redução das áreas de cultivo e da produção de forragem de palma forrageira dos cultivares Gigante, Redonda e o clone IPA 20, suscetíveis ao inseto (Souza et al., 2010).

Palma Miúda [Gênero: Nopalea sp., (Espécie: Nopalea cochenilifera Salm-Dyck)]. O genótipo Miúda é um dos principais cultivados, principalmente por apresentar resistência à cochonilha do carmim Dactylopius opuntiae. É considerada altamente palatável por ruminantes em comparação com outras variedades, porém apresenta uma menor resistência à seca (Silva \& Santos, 2006). Segundo Silva \& 
Sampaio (2015) são plantas de porte pequeno e caule bastante ramificado. Sua raquete pesa cerca de 350 $\mathrm{g}$, possuem quase $25 \mathrm{~cm}$ de comprimento, forma acentuadamente obovada (ápice mais largo que a base) e coloração verde intenso brilhante. É uma variedade mais exigente em fertilidade de solo e sensível a cochonilha de escama (Cavalcanti et al., 2008a; Silva \& Santos, 2006). É mais exigente em umidade e temperatura noturna mais amena quando comparada aos outros cultivares (Donato et al., 2014).

Palma Orelha de Elefante Mexicana - OEM [Gênero: Opuntia sp. (espécie: Opuntia strica Haw.)]. Possui como principal característica resistência à infestação por cochonilha do carmim (Vasconcelos et al., 2009). A OEM apresenta espinhos, o que pode comprometer sua palatabilidade e dificultar seu manejo como planta forrageira (Cavalcanti et al., 2008b). Entretanto, a palma OEM tem mostrado boas repostas agronômicas, menos exigente em nutrientes, mais tolerante às condições de estresse hídrico e também tem apresentado maior produção de matéria seca por unidade de área do que a palma Miúda. A variedade tem se destacado nas recentes pesquisas no que se diz respeito aos efeitos na produção animal. Aparentemente, alguns fatores que conferem a resistência às pragas, como lignificação, silicificação ou células epidérmicas mais espessas, não influenciam negativamente o valor nutritivo dos genótipos. Visto que, genótipos de palma forrageira resistentes à cochonilha-do-carmim mostram características nutricionais semelhantes ou melhores do que genótipos suscetíveis tradicionalmente usados (Santos et al., 2018).

\section{Composição bromatológica}

Independente do gênero (Opuntia ou Nopalea), os principais genótipos de palma forrageira pesquisados para nutrição animal apresentam composição química com pequena variação (Tabela 1). Com médias de $110 \pm 27 \mathrm{~g} / \mathrm{kg}$ de Matéria Seca (MS), 874 $\pm 42 \mathrm{~g} / \mathrm{kg}$ de Matéria Orgânica (MO), $126 \pm 42 \mathrm{~g} / \mathrm{kg}$ de Matéria Mineral (MM), $48 \pm 12 \mathrm{~g} / \mathrm{kg}$ de Proteína Bruta (PB), $16 \pm 5 \mathrm{~g} / \mathrm{kg}$ de Extrato Etéreo (EE), $536 \pm 72 \mathrm{~g} / \mathrm{kg}$ de Carboidratos Não-Fibrosos (CNF), $256 \pm 27 \mathrm{~g} / \mathrm{kg}$ de Fibra Insolúvel em Detergente Neutro (FDN) e 169 $\pm 39 \mathrm{~g} / \mathrm{kg}$ de Fibra Insolúvel em Detergente Ácido (FDA).

Tabela 1. Matéria seca (MS), matéria orgânica (MO), proteína bruta (PB), carboidratos não fibrosos (CNF), fibra em detergente neutro (FDN) e fibra em detergente ácido (FDA) de diferentes espécies e genótipos de palma forrageira

\begin{tabular}{|c|c|c|c|c|c|c|c|c|c|}
\hline Referências & Palma Forrageira & $\begin{array}{l}\mathrm{MS} \\
\mathrm{g} / \mathrm{kg}\end{array}$ & $\begin{array}{l}\mathrm{MO} \\
\mathrm{g} / \mathrm{kg}\end{array}$ & $\begin{array}{l}\mathrm{MM} \\
\mathrm{g} / \mathrm{kg}\end{array}$ & $\begin{array}{l}\mathrm{PB} \\
\mathrm{g} / \mathrm{kg}\end{array}$ & $\begin{array}{c}\mathrm{EE} \\
\mathrm{g} / \mathrm{kg}\end{array}$ & $\begin{array}{l}\mathrm{CNF} \\
\mathrm{g} / \mathrm{kg}\end{array}$ & $\begin{array}{c}\text { FDN } \\
\mathrm{g} / \mathrm{kg}\end{array}$ & $\begin{array}{l}\text { FDA } \\
\mathrm{g} / \mathrm{kg}\end{array}$ \\
\hline$\overline{\text { Silva et al. (2018) }}$ & Nopalea cochenillifera Salm-Dyck ${ }^{2}$ & 94 & 881 & 119 & 60 & 12 & 547 & 262 & - \\
\hline Monteiro et al. (2019) & Nopalea cochenillifera Salm-Dyck ${ }^{2}$ & 150 & 910 & 90 & 35 & - & 574 & 257 & - \\
\hline Oliveira et al. (2018) & Nopalea cochenillifera Salm-Dyck ${ }^{2}$ & 109 & 844 & 156 & 44 & 14 & 534 & 243 & 146 \\
\hline Siqueira et al. (2018) & Nopalea cochenillifera Salm-Dyck ${ }^{2}$ & 139 & 860 & 140 & 41 & 15 & 523 & 277 & - \\
\hline Silva et al. (2017) & Nopalea cochenillifera Salm-Dyck ${ }^{2}$ & 140 & 913 & 87 & 32 & 11 & 667 & 202 & 200 \\
\hline Lopes et al. (2017) & Nopalea cochenillifera Salm-Dyck ${ }^{2}$ & 83 & 789 & 211 & 75 & 18 & 460 & 236 & - \\
\hline Silva et al. (2018) & Opuntia stricta Haw $^{1}$ & 157 & 929 & 71 & 33 & 10 & 639 & 246 & - \\
\hline Monteiro et al. (2019) & Opuntia stricta Haw $^{1}$ & 123 & 914 & 86 & 55 & - & 550 & 259 & - \\
\hline Conceição et al. (2018) & Opuntia stricta Haw $^{1}$ & 105 & 802 & 198 & 55 & 12 & 406 & 291 & - \\
\hline Barros et al. (2018) & Opuntia stricta $\mathrm{Haw}^{1}$ & 112 & 869 & 131 & 51 & 16 & 528 & 257 & - \\
\hline Silva et al. (2018) & Opuntia stricta $\mathrm{Haw}^{1}$ & 127 & 914 & 86 & 33 & 12 & 642 & 227 & 172 \\
\hline Pessoa et al. (2013) & Opuntia ficus indica Mill $^{3}$ & 93 & 880 & 120 & 38 & 24 & 527 & 290 & 225 \\
\hline Batista et al. (2009) & Opuntia ficus indica Mill $^{3}$ & 153 & 919 & 81 & 44 & 24 & 398 & 248 & 179 \\
\hline \multirow[t]{3}{*}{ Bispo et al. (2007) } & Opuntia ficus indica Mill $^{3}$ & 93 & 882 & 118 & 49 & 24 & 480 & 328 & 241 \\
\hline & Opuntia ficus indica Mill $^{3}$ & 82 & 925 & 75 & 46 & 22 & 614 & 244 & 130 \\
\hline & Nopalea cochenillifera Salm-Dyck2 & 96 & 848 & 152 & 46 & 17 & 539 & 246 & 128 \\
\hline \multirow[t]{3}{*}{ Rocha Filho (2012) } & Nopalea cochenillifera Salm-Dyck4 & 74 & 815 & 185 & 59 & 16 & 504 & 236 & 140 \\
\hline & Opuntia stricta $\mathrm{Haw}^{1}$ & 77 & 859 & 141 & 69 & 19 & 509 & 262 & 139 \\
\hline & Opuntia undulata Griffiths ${ }^{5}$ & 82 & 860 & 140 & 51 & 10 & 549 & 250 & 155 \\
\hline
\end{tabular}

${ }^{1} \mathrm{cv}$. Orelha de Elefante Mexicana; ${ }^{2} \mathrm{cv}$. Miúda; ${ }^{3} \mathrm{cv}$. Gigante; ${ }^{4} \mathrm{cv}$. IPA Sertânia; ${ }^{5} \mathrm{cv}$. Orelha de Elefante Africana. 
Uma das principais características nutricionais da palma forrageira é o alto teor de CNF e baixo teor de lignina, os quais resultam em alta degradabilidade ruminal da MS (Batista et al., 2009). Isso desperta o interesse para sua utilização em substituição a concentrados energéticos, como o milho ou trigo (Conceição et al., 2018; Costa et al., 2012; Felix et al., 2016). Além disso, vale ressaltar que uma porção elevada do CNF é composta por amido, $200 \mathrm{~g} / \mathrm{kg}$ MS em média (Batista et al., 2009) e pectina $72 \mathrm{~g} / \mathrm{kg}$ MS em média (Batista et al., 2003a), que é considerada fibra solúvel, sua fermentação produz menos lactato, reduzindo o risco de acidose ruminal.

O teor de FDN da palma é uma característica peculiar em relação a outras fontes de volumoso (Rocha Filho, 2012), sendo considerado de baixa efetividade. Tornando-se necessário à sua associação com outra forragem, para manter os níveis de fibra dentro do recomendável (Ferreira et al., 2009). Segundo É importante ressaltar a necessidade de se adicionar um mínimo de 15 a $20 \%$ de uma fonte de fibra fisicamente efetiva, na base da MS, para

otimizar o consumo de MS em dietas baseadas em palma forrageira. A substituição total da fonte de fibra efetiva por palma na dieta já foi relatada por diversos autores, onde todos os resultados comprovam decréscimo (linear ou quadrático) do CMS com o nível mais alto de substituição da fonte de fibra efetiva (Barros et al., 2018; Oliveira et al., 2017).

O teor de PB da palma é insuficiente para o adequado desempenho animal. Entretanto, a alta concentração de carboidratos solúveis na palma possibilita a incorporação de fontes de nitrogênio não proteico (NNP) com o objetivo de elevar o teor de proteína (Ferreira et al., 2009), sendo comum a associação com ureia pecuária. A proporção de proteína verdadeira é baixa, segundo Batista et al. (2003b), as diversas variedades de palma continham em média altos níveis de proteína solúvel e baixos níveis de proteína insolúvel em detergente neutro e ácido, 57\% da proteína bruta na palma forrageira é representado por PB solúvel, sendo que $71 \%$ dessa fração é nitrogênio não proteico (NNP). Com relação ao EE, apesar do baixo teor, Abidi et al. (2009) relataram alta concentração de ácidos graxos poliinsaturados em $67,7 \%$ do total de ácidos graxos presentes na palma forrageira. Aliado a isso, a rápida taxa de passagem pode favorecer a diminuição da biohidrogenação no rúmen, o que pode promover mudança desejável no perfil de ácidos graxos da carne e do leite de animais alimentados com palma forrageira.

\section{Consumo e digestibilidade}

A palma forrageira apresenta alta aceitabilidade e grandes quantidades podem ser voluntariamente consumidas (Ferreira et al., 2009). Entretanto, devido a diferenças na composição química ou morfológica, como presença de espinhos, pode haver diferença entre espécies no efeito sobre a ingestão da ração, como também o nível de inclusão de palma na dieta, a participação de outros alimentos na ração, ou a associação entre esses fatores, contribuam para essas diferenças (Batista et al., 2009). Siqueira et al. (2017) avaliaram a inclusão de palma forrageira cv. Miúda em substituição ao feno de tifton para novilhos e relataram comportamento quadrático para o consumo de matéria seca (CMS) e matéria orgânica digestível (MOD), com valores máximos estimados de 8,89 e 5,75 kg/dia com inclusão máxima de palma em 339 e 418 g/kg, respectivamente. Em adição, Barros et al. (2018) avaliando a substituição total do feno tifton por palma (OEM) na dieta de novilhas e encontraram decréscimo linear no consumo de MS de 4,5 para 3,65 kg/dia, respectivamente. Corroborando com Silva et al. (2018), ao substituir palma Miúda por OEM para vacas leiteiras, houve decréscimo linear de 14,3 para 12,9 kg/dia no CMS, como também, 9,38 para 7,92 kg/dia de nutrientes digestíveis totais (NDT). Por outro lado, Monteiro et al. (2019) relatam que é possível substituir 100\% de Miúda pelo genótipo OEM para vacas leiteiras de alta produção, sem prejuízo no consumo de MS e demais nutrientes, destacando o genótipo OEM como uma nova opção de forragem para pequenos sistemas pecuários em regiões semiáridas, proporcionando diversificação agrícola.

Oliveira et al. (2017) em estudo com a substituição total da fonte volumosa, cana-de-açúcar por palma forrageira cv. Miúda, para ovinos em crescimento, verificou decréscimo linear no consumo de 1,10 para $0,97 \mathrm{~kg} / \mathrm{dia}$. Costa et al. (2012) relataram os efeitos da substituição de milho por palma forrageira cv. Gigante no desempenho de cordeiros, sendo o máximo CMS foi de aproximadamente $54,0 \%$ de palma em substituição na dieta, onde a ingestão de MS alcançou 1,49 kg/dia, como também, efeito quadrático para o consumo de NDT, estimado como máximo 0,904 kg/dia com 43,3\% de palma. 
Não foi observado efeito na ingestão de cordeiros alimentados com palma forrageira cv. Miúda substituindo farelo de trigo (Felix et al., 2016). Pessoa et al. (2013) relataram que diferentes suplementos (farelo de trigo, farelo de soja, farelo de algodão ou caroço de algodão) associados à palma forrageira cv. Gigante em dietas para ovinos não alterou o CMS.

Quando a palma forrageira é utilizada como base da dieta, também se pode observar comportamentos distintos, Lopes et al. (2017) avaliando a substituição do feno de tifton por feno de alfafa em dietas com 400 g/kg de MS de palma Miúda para cabras leiteiras, relataram aumento do CMS, assim como, Souza et al. (2009) avaliando a substituição do feno de tifton por casca de soja na dieta de caprinos com 600 $\mathrm{g} / \mathrm{kg}$ de palma Gigante, relataram diminuição linear na ingestão de matéria seca. Entretanto, Ramos et al. (2013) associando diferentes fontes de fibra como feno de tifton, casca de soja e caroço de algodão à palma forrageira, relatam não influenciar o consumo de MS no presente estudo. É importante ressaltar também que, de acordo com Mertens (1994) e Maggioni et al. (2009), o aumento no consumo de alimentos por um animal geralmente provoca um aumento na taxa de passagem da digesta, e assim, o alimento é exposto à ação digestiva por um curto período de tempo e a digestibilidade pode ser reduzida.

Na Tabela 2 são apresentados os dados referentes à digestibilidade aparente da palma forrageira. De acordo com os dados da literatura citada, em média a digestibilidade da matéria seca (DMS) foi de 658 $\pm 78 \mathrm{~g} / \mathrm{kg}$, matéria orgânica (DMO) $699 \pm 57 \mathrm{~g} / \mathrm{kg}$; proteína bruta (DPB) $705 \pm 121 \mathrm{~g} / \mathrm{kg}$; carboidratos não fibrosos (DCNF) $909 \pm 38 \mathrm{~g} / \mathrm{kg}$ e da fibra em detergente neutro (DFDN) $509 \pm 64 \mathrm{~g} / \mathrm{kg}$; em função de diferentes espécies e genótipos de palma forrageira.

Tabela 2. Digestibilidade aparente da matéria seca (DMS), matéria orgânica (DMO), proteína bruta (DPB), carboidratos não fibrosos (DCNF) e da fibra em detergente neutro (DFDN) de diferentes espécies e genótipos de palma forrageira

\begin{tabular}{|c|c|c|c|c|c|c|c|c|c|}
\hline Referência & Animal & Palma Forrageira & $\begin{array}{c}\text { Palma } \\
\mathrm{g} / \mathrm{kg}\end{array}$ & $\begin{array}{c}\text { Volumoso } \\
\mathrm{g} / \mathrm{kg}\end{array}$ & $\begin{array}{l}\text { DMS } \\
\mathrm{g} / \mathrm{kg}\end{array}$ & $\begin{array}{l}\text { DMO } \\
\mathrm{g} / \mathrm{kg}\end{array}$ & $\begin{array}{l}\text { DPB } \\
\mathrm{g} / \mathrm{kg}\end{array}$ & $\begin{array}{c}\text { DCNF } \\
\mathrm{g} / \mathrm{kg}\end{array}$ & $\begin{array}{c}\text { DFDN } \\
\mathrm{g} / \mathrm{kg}\end{array}$ \\
\hline Cardoso et al. (2019) & Ovino & Nopalea cochenillifera Salm-Dyck ${ }^{2}$ & 450 & 50 & 755 & 793 & 759 & 917 & 503 \\
\hline Siqueira et al. (2017) & Bovino & Nopalea cochenillifera Salm-Dyck ${ }^{2}$ & 588 & 90 & 788 & 789 & 832 & - & 597 \\
\hline Lopes et al. (2017) & Caprino & Nopalea cochenillifera Salm-Dyck ${ }^{2}$ & 400 & 300 & 628 & 635 & 684 & - & 596 \\
\hline Lins et al. (2016) & vino & Nopalea cochenillifera Salm-Dyck ${ }^{2}$ & 492 & 377 & 666 & 700 & 737 & 861 & 519 \\
\hline \multirow{2}{*}{ Silva et al. (2018) } & \multirow{2}{*}{ Bovino } & Nopalea cochenillifera Salm-Dyck ${ }^{2}$ & 480 & 300 & 716 & 747 & 863 & - & 529 \\
\hline & & Opuntia stricta $\mathrm{Haw}^{1}$ & 480 & 300 & 658 & 704 & 845 & - & 495 \\
\hline Monteiro et al. (2019) & Bovino & Opuntia stricta Haw $^{1}$ & 390 & 310 & 686 & 700 & 764 & 964 & 387 \\
\hline Silva et al. (2017) & Bovino & Opuntia stricta Haw $^{1}$ & 500 & 300 & 673 & 727 & 656 & - & 622 \\
\hline Barros et al. (2018) & Bovino & Opuntia stricta Haw $^{1}$ & 500 & 0 & 726 & 727 & 644 & 860 & 485 \\
\hline Pessoa et al. (2013) & Ovino & Opuntia ficus indica Mill $^{3}$ & 628 & 251 & 598 & 660 & 777 & 872 & 437 \\
\hline Santos et al. (2010) & Ovino & Opuntia ficus indica Mill $^{3}$ & 730 & 150 & 679 & 728 & 709 & - & 613 \\
\hline Vieira et al. (2008) & Caprino & Opuntia ficus indica Mill $^{3}$ & 765 & 50 & 700 & 740 & 707 & 870 & 550 \\
\hline Bispo et al. (2007) & Ovino & Opuntia ficus indica Mill $^{3}$ & 560 & 90 & 695 & 715 & 708 & 881 & 526 \\
\hline Misra et al. (2006) & Ovino & Opuntia ficus indica Mill $^{3}$ & 309 & ad lib. & 463 & - & 315 & - & 452 \\
\hline Ben Salem et al. (1996) & Ovino & Opuntia ficus indica Inermis & 300 & ad lib. & 491 & 543 & 537 & - & 483 \\
\hline \multirow{5}{*}{ Rocha Filho (2012) } & Ovino & Opuntia ficus indica Mill $^{3}$ & 400 & 585 & 631 & 660 & 686 & 948 & 423 \\
\hline & Ovino & Nopalea cochenillifera Salm-Dyck ${ }^{2}$ & 400 & 585 & 664 & 691 & 703 & 934 & 506 \\
\hline & Ovino & Nopalea cochenillifera Salm-Dyck ${ }^{4}$ & 400 & 585 & 662 & 689 & 728 & 934 & 511 \\
\hline & Ovino & Opuntia stricta Haw $^{1}$ & 400 & 585 & 658 & 681 & 733 & 916 & 508 \\
\hline & Ovino & Opuntia undulata Griffiths $^{5}$ & 400 & 585 & 627 & 656 & 705 & 945 & 437 \\
\hline
\end{tabular}

${ }^{1}$ cv. Orelha de Elefante Mexicana; ${ }^{2}$ cv. Miúda; ${ }^{3}$ cv. Gigante; ${ }^{4}$ cv. IPA Sertânia; ${ }^{5}$ cv. Orelha de Elefante Africana.

Todas as espécies de palma forrageira apresentam alta digestibilidade da MS, com pequena diferença entre elas (Batista et al., 2009), como também MO, PB e CNF. Diversos autores relatam aumento da digestibilidade da MS e/ou demais nutrientes (MO, PB, EE, CNF e CHTO) da dieta com a inclusão de palma forrageira (Bispo et al., 2007; Conceição et al., 2016; Costa et al., 2012; Felix et al., 2016; Lins et al., 2016; Monteiro et al., 2014; Siqueira et al., 2017). 
Os coeficientes máximos de digestibilidade da MO, PB, EE, CNF e CHTO da palma forrageira podem ser encontrados em diferentes níveis de inclusão e dependente da associação com demais ingredientes e categoria animal. Conforme relatado por Mertens (1994) a digestibilidade de um alimento está intimamente relacionada com a composição química, sendo a fração fibrosa que exerce maior influência, tanto em quantidade como em qualidade, além disso, muitos outros fatores podem influenciar na digestibilidade, nível de consumo, efeito associativo, proporção dos ingredientes, processamento dos alimentos, além de outros fatores dependentes dos animais e do nível nutricional. Rocha Filho (2012) verificou que DMS de diferentes genótipos foram semelhantes para ovinos, assim como Monteiro et al. (2019) substituindo palma Miúda por OEM para vacas em lactação. Ferreira et al. (2009) relatam a palma forrageira como muito digestível, apresentando coeficientes de digestibilidade in vitro na matéria seca da ordem de 74,4; 75,0 e 77,40\% para as cultivares redonda, Gigante e Miúda, respectivamente. Entretanto, para os nutrientes PB e FDN, quando comparadas a digestibilidade das diferentes espécies, em função das distintas pesquisas, nota-se maiores médias para Nopalea Miúda (DPB $797 \pm 88 \mathrm{~g} / \mathrm{kg}$; DFDN $558 \pm 39 \mathrm{~g} / \mathrm{kg}$ ), seguido das espécies do gênero Opuntia OEM (DPB $728 \pm 83 \mathrm{~g} / \mathrm{kg}$; DFDN $499 \pm$ $84 \mathrm{~g} / \mathrm{kg}$ ) e Gigante (DPB $650 \pm 167 \mathrm{~g} / \mathrm{kg}$; DFDN $500 \pm 75 \mathrm{~g} / \mathrm{kg}$ ). Silva et al. (2018) relataram diminuição da DPB avaliando a substituição de palma Miúda por OEM na dieta de vacas em lactação. Assim como, Rocha Filho (2012) avaliando os efeitos dos diferentes genótipos de palma sobre o coeficiente de DPB para vacas em lactação confirmou superioridade da DPB para palma Miúda em comparação a Gigante. O mesmo autor, em pesquisa com ovinos, relatou maior DPB para palma OEM em comparação a Gigante.

É valido ressaltar que aliado ao baixo teor de fibra efetiva em sua composição, a inclusão de palma na alimentação de ruminantes pode diminuir a DFDN da dieta. A associação de palma com concentrados elevar ao máximo a degradabilidade de CNF no rúmen, que pode favorecer a produção de ácidos graxos voláteis, entretanto, como também a mucilagem pode reduzir sua a absorção, e o acumulo diminuir o $\mathrm{pH}$ ruminal, resultando em ambiente desfavorável ao crescimento dos microrganismos que degradam a referida fração, aliado ao aumento de taxa de passagem, e diminuição do tempo de colonização e degradação. Esses fatores associados podem resultar em efeitos prejudiciais a atividade fibrolítica microbiana.

A literatura também confirma que em comparação, os diferentes genótipos de palma também podem divergir quanto a DFDN. Monteiro et al. (2019) relataram que a DFDN aumentou linearmente com a substituição da Miúda por OEM. Rocha Filho (2012) relatou superioridade na DFDN para os genótipos Miúda, OEM e IPA Sertânia em comparação a palma Gigante em dietas para ovinos como também para vacas leiteiras. Monteiro et al. (2014), ao substituir farelo de trigo por palma (Gigante) na dieta de novilhas, a DFDN diminuiu linearmente. Barros et al. (2018) explicam que a redução na DFDN pode ser associada pelo aumento do conteúdo da CNF e pela diminuição da fibra, com a substituição de tifton por palma (OEM). Corroborando também com Cavalcanti et al. (2006) que relataram a redução da DFDN devido a altos níveis de carboidratos solúveis no ambiente ruminal, avaliando o efeito da substituição do feno de Tifton por palma forrageira (Gigante).

Por fim, de acordo com Fotius et al. (2014) diferentes sequências de oferta dos ingredientes em dietas com palma forrageira, não afetam o consumo de nutrientes, contudo, a sequência da oferta de palma e concentrado pela manhã e feno e concentrado a tarde, independentemente do horário de fornecimento dos ingredientes, promove melhor digestibilidade dos nutrientes (MS, MO, FDN e CHOT).

\section{Degradabilidade ruminal}

De acordo com Batista et al. (2009), todos os cultivares de palma forrageira apresentaram alta degradabilidade ruminal da MS, com poucas diferenças entre eles. Batista et al. (2003a) relataram desaparecimento in situ da MS de mais de $800 \mathrm{~g} / \mathrm{kg}^{-1} \mathrm{em}$ até $48 \mathrm{~h}$ de incubação, justificando isso ao baixo teor de fibras e alto teor de carboidratos solúveis. Ainda os mesmos autores, relaram diferenças entre as variedades de palma avaliadas e descrevem ser decorrentes principalmente de diferentes padrões de fermentação dos carboidratos. Batista et al. (2003b) relataram que 63\% da FDN em média dos genótipos avaliados de palma forrageira desapareceram após $48 \mathrm{~h}$ de incubação ruminal in situ. Alta taxa de degradação na FDN pode ser atribuída à baixa concentração de lignina, $11,6 \mathrm{~g} / \mathrm{kg}^{-1} \mathrm{em}$ média (Batista et al., 2009). Ainda os mesmos autores relatam que as diferenças encontradas entre os estudos são 
provavelmente devido a diferenças nos materiais utilizados nas incubações in situ e a forma de processamento da palma. Segundo Batista et al. (2003b), as variedades de palma apresentaram baixa fração solúvel, que variou de 17 a $111 \mathrm{~g} / \mathrm{kg}^{-1}$ de PB. Isto é diferente de outras forragens, como leguminosas e fenos de gramíneas, onde a proteína solúvel constitui uma grande porção da PB total. A fração lentamente degradável $\left(\mathrm{g} / \mathrm{kg}^{1}\right.$ de $\left.\mathrm{PB}\right)$ e sua taxa de degradação foram semelhantes entre as variedades de palma e apresentaram uma média de $900 \mathrm{~g} / \mathrm{kg}^{-1}$ de PB e $9 \% / \mathrm{h}^{-1}$, respectivamente.

A alta degradabilidade ruminal tem sido destacada por maximizar a capacidade fermentativa do rúmen, aumentando a síntese de proteína microbiana, a produção de ácidos graxos voláteis e, com consequência, a condução de nutrientes para o animal (Berchielli et al., 2011). Contudo, em altos níveis de palma forrageira na alimentação de ruminantes, a alta degradabilidade ruminal, também é relatada por causar em distúrbios digestivos, como enchimento e efeito laxativo (Gebremariam et al., 2006).

\section{Oxalatos}

É relatado que a palma forrageira possui elevados teores de Ca, além de $\mathrm{Ke} \mathrm{Mg}$ (Batista et al., 2009), o que pode reduzir a absorção desses minerais, limitando o crescimento microbiano e a digestibilidade de diferentes ingredientes (Ben Salem et al., 1996). Rekik et al. (2010) relataram teor de 105,3 g/kg MS de oxalato, o que pode tornar reduzida disponibilidade de cálcio, e outros minerais, como resultado do seu sequestro na forma de cristais de oxalato de cálcio (McConn \& Nakata, 2004).

O ácido oxálico é degradado no rúmen, por bactérias ruminais (Oxalobacter formigenes) com objetivo de obter energia, que transforma oxalato em ácido fórmico e CO2 (Allison et al., 1985), disponibilizando o cálcio para absorção. Para suprir as necessidades de crescimento desta população de bactérias, seriam necessárias grandes quantidades de oxalato, visto que, este metabolismo gera proporcionalmente pouca energia. Dessa maneira, há uma população bastante pequena de bactérias dessa espécie no rúmen, que podem metabolizar o oxalato ingerido (Weimer, 1998). A absorção de oxalato provoca elevações na excreção urinária deste composto, o que pode aumentar a precipitação de oxalato de cálcio e a formação de cálculos urinários. Segundo McConn \& Nakata (2004) $75 \%$ dos cálculos urinários e renais contêm como principal componente oxalato de cálcio. Está bem estabelecido que o ácido oxálico apresenta grande afinidade com o cálcio e o magnésio, dificultando sua utilização pelo hospedeiro e microrganismos ruminais, de tal maneira, essa questão deve ser considerada durante a formulação de dietas com palma para ruminantes (Ben Salem \& Abidi, 2007).

\section{Conclusão}

A utilização de palma forrageira já é uma realidade na alimentação de ruminantes em diversas regiões carentes de recursos hídricos em várias regiões do mundo, e já foi documentado sendo apontada como alternativa a diversos alimentos convencionais empregados na nutrição animal. Assim, o entendimento do valor nutritivo dos diferentes genótipos desta forragem é necessário para otimizar a nutrição de ruminantes e maximizar as respostas de desempenho produtivo.

\section{Referências bibliográficas}

Abidi, S., Ben Salem, H., Vasta, V. \& Priolo, A. (2009). Supplementation with barley or spineless cactus (Opuntia ficus indica $\mathrm{f}$. inermis) cladodes on digestion, growth and intramuscular fatty acid composition in sheep and goats receiving oaten hay. Small Ruminant Research, 87(1-3):9-16.

Allison, M. J., Dawson, K. A., Mayberry, W. R. \& Foss, J. G. (1985). Oxalobacter formigenes gen. nov., sp. nov.: oxalate-degrading anaerobes that inhabit the gastrointestinal tract. Archives of Microbiology, 141(1):1-7.

Barbosa, M. L., Silva, T. G. F., Zolnier, S. \& Ferreira, W. P. M. (2018). Environmental variables influencing the expression of morphological characteristics in clones of the forage cactus. Revista Ciência Agronômica, 49(3):399-408.

Barros, L. J. A., Ferreira, M. A., Oliveira, J. C. V., Santos, D. C., Chagas, J. C. C., Alves, A. M. S. V., ... Freitas, W. R. (2018). Replacement of Tifton hay by spineless cactus in Girolando post-weaned heifers diets. Tropical Animal Health and Production, 50(1):149-154. 
Batista, A. M., Mustafa, A. F., McAllister, T., Wang, Y., Soita, H. \& McKinnon, J. J. (2003a). Effects of variety on chemical composition, in situ nutrient disappearance and in vitro gas production of spineless cacti. Journal of the Science of Food and Agriculture, 83(5):440-445.

Batista, A. M. V., Mustafa, A. F., Santos, G. R. A., Carvalho, F. F. R., Dubeux Júnior, J. C., Lira, M. A. \& Barbosa, S. B. P. (2003b). Chemical composition and ruminal dry matter and crude protein degradability of spineless cactus. Journal of Agronomy and crop Science, 189(2):123-126.

Batista, Â. M. V., Ribeironeto, A. C., Lucena, R. B., Santos, D. C., Dubeux Junior, J. \& Mustafa, A. F. (2009). Chemical composition and ruminal degradability of spineless cactus grown in Northeastern Brazil. Rangeland Ecology \& Management, 62(3):297-301.

Ben Salem, H. \& Abidi, S. (2007). Recent advances on the potential use of Opuntia spp. in livestock feeding. Acta Horticulturae, 811317-326.

Ben Salem, H., Nefzaoui, A., Abdouli, H. \& Orskov, E. R. (1996). Effect of increasing level of spineless cactus (Opuntia ficus indica var inermis) on intake and digestion by sheep given straw-based diets. Animal Science, 62293-299.

Ben Salem, H. \& Smith, T. (2008). Feeding strategies to increase small ruminant production in dry environments. Small Ruminant Research, 77(2-3):174-194.

Berchielli, T. T., Pires, A. V. \& Oliveira, S. G. (2011). Nutrição de Ruminantes. Jaboticabal, Brazil: FUNEP.

Bispo, S. V., Ferreira, M. A., Véras, A. S. C., Batista, A. M. V., Pessoa, R. A. S. \& Bleuel, M. P. (2007). Palma forrageira em substituição ao feno de capim-elefante. Efeito sobre consumo, digestibilidade e características de fermentação ruminal em ovinos. Revista Brasileira de Zootecnia, 36(6):1902-1909.

Cardoso, D. B., Carvalho, F. F. R., Medeiros, G. R., Guim, A., Cabral, A. M. D., Véras, R. M. L., .. . Nascimento, A. G. O. (2019). Levels of inclusion of spineless cactus (Nopalea cochenillifera Salm Dyck) in the diet of lambs. Animal Feed Science and Technology, 24723-31.

Cavalcanti, C. V. A., Ferreira, M. A., Carvalho, M. C., Veras, A. S. C., Lima, L. E. \& Silva, F. M. (2006). Forage cactus (Opuntia ficus indica Mill) and urea in replacement of tifton hay (Cynodon spp.) in lactating Holstein cows diet. 1. Digestibility. Acta Scientiarum-Animal Sciences, 28(2):145-152.

Cavalcanti, C. V. A., Ferreira, M. d. A., Carvalho, M. C. U., Véras, A. S. C., Silva, F. M. d. U. \& Lima, L. E. d. I. (2008a). Palma forrageira enriquecida com ureia em substituição ao feno de capim-tifton 85 em rações para vacas da raça Holandesa em lactação. Revista Brasileira de Zootecnia, 37689-693.

Cavalcanti, M. C. A., Batista, Â. M. V., Guim, A., Andrade, M. L., Ribeiro, V. L. \& Ribeiro Neto, A. C. (2008b). Consumo e comportamento ingestivo de caprinos e ovinos alimentados com palma gigante (Opuntia ficus-indica Mill) e palma orelha-de-elefante (Opuntia sp.). Acta Scientiarum. Animal Sciences, 30(2):173-179.

Conceição, M. G., Ferreira, M. A., Silva, J. L., Costa, C. T. F., Chagas, J. C. C. \& Monteiro, C. C. F. (2018). Can cactus (Opuntia stricta [Haw.] Haw) cladodes plus urea replace wheat bran in steers' diet? Asian-Australasian journal of animal sciences, 31(10):1627-1634.

Conceição, M. G., Ferreira, M. d. A., Campos, J. M. S., Silva, J. L., Detmann, E., Siqueira, M. C. B., . . . Costa, C. T. F. (2016). Replacement of wheat bran with spineless cactus in sugarcane-based diets for steers. Revista Brasileira de Zootecnia, 45(4):158-164.

Costa, R. G., Treviño, I. H., Medeiros, G. R., Medeiros, A. N., Pinto, T. F. \& Oliveira, R. L. (2012). Effects of replacing corn with cactus pear (Opuntia ficus indica Mill) on the performance of Santa Inês lambs. Small Ruminant Research, 102(1):13-17.

Donato, P. E. R., Pires, A. J. V., Donato, S. L. R., Bonomo, P., Silva, J. A. \& Aquino, A. A. (2014). Morfometria e rendimento da palma forrageira 'Gigante'sob diferentes espaçamentos e doses de adubação orgânica. Revista Brasileira de Ciências Agrárias, 9(1):151-158.

Dubeux Júnior, J. C. B., Araújo Filho, J. T., Santos, M. V. F., Lira, M. A., Santos, D. C. \& Pessoa, R. A. S. (2013). Potential of cactus pear in South America. Cactusnet Newsletter, 1329-40.

Felix, S. C. R., Pessoa, R. A. S., Ferreira, M. A., Soares, L. F. P., Silva, J. L., Abreu, K. S. F. \& Melo, A. C. C. (2016). Intake, performance, and carcass characteristics of lambs fed spineless cactus replacing wheat bran. Tropical Animal Health and Production, 48(2):465-468. 
Ferreira, M. A., Silva, F. M., Bispo, S. V. \& Azevedo, M. (2009). Estratégias na suplementação de vacas leiteiras no semi-árido do Brasil. Revista Brasileira de Zootecnia, 38322-329.

Fotius, A. C. A., Ferreira, M. A., Véras, A. S. C., Salla, L. E., Souza, A. R. D. L. \& Bispo, S. V. (2014). Estratégia de nutrientes para ovinos em distintas sequências de fornecimento alimentar em dieta a base de palma forrageira. Revista Brasileira de Saúde e Produção Animal, 15(2):504-516.

Gebremariam, T., Melaku, S. \& Yami, A. (2006). Effect of different levels of cactus (Opuntia ficusindica) inclusion on feed intake, digestibility and body weight gain in tef (Eragrostis tef) straw-based feeding of sheep. Animal Feed Science and Technology, 131(1-2):42-51.

Lins, S. E. B., Pessoa, R. A. S., Ferreira, M. d. A., Campos, J. M. d. S., Silva, J. A. B. A., Silva, J. d. L., . . Melo, T. T. d. B. (2016). Spineless cactus as a replacement for wheat bran in sugar cane-based diets for sheep: intake, digestibility, and ruminal parameters. Revista Brasileira de Zootecnia, 45(1):26-31.

Lopes, L. A., Carvalho, F. F. R., Cabral, A. M. D., Batista, Â. M. V., Camargo, K. S., Silva, J. R. C., . . . Silva, J. (2017). Replacement of tifton hay with alfalfa hay in diets containing spineless cactus (Nopalea cochenillifera Salm-Dyck) for dairy goats. Small Ruminant Research, 1567-11.

Maggioni, D., Marques, J. A., Rotta, P. P., Zawadzki, F., Ito, R. H. \& Prado, I. N. (2009). Ingestão de alimentos. Semina: Ciências Agrárias, 30(4):963-974.

Marques, O. F. C., Paula Gomes, L. S., Mourthé, M. H. F., Santos, T. G. B. \& Pires Neto, O. S. (2017). Palma forrageira: cultivo e utilização na alimentação de bovinos. Caderno de Ciências Agrárias, 9(1):75-93.

McConn, M. M. \& Nakata, P. A. (2004). Oxalate reduces calcium availability in the pads of the prickly pear cactus through formation of calcium oxalate crystals. Journal of Agricultural and Food Chemistry, 52(5):1371-1374.

Mertens, D. R. (1994). Regulation of Forage Intake. In J. R. Fahey (Ed.), Forage Quality, Evaluation, and Utilization (pp. 450-493). Madison, WI, USA: American Society of Agronomy.

Misra, A. K., Mishra, A. S., Tripathi, M. K., Chaturvedi, O. H., Vaithiyanathan, S., Prasad, R. \& Jakhmola, R. C. (2006). Intake, digestion and microbial protein synthesis in sheep on hay supplemented with prickly pear cactus [Opuntia ficus-indica (L.) Mill.] with or without groundnut meal. Small Ruminant Research, 63(1-2):125-134.

Monteiro, C. C. F., Ferreira, M. A., Véras, A. S. C., Guido, S. I., Almeida, M. P., Silva, R. C. \& Inácio, J. G. (2019). A new cactus variety for dairy cows in areas infested with Dactylopius opuntiae. Animal Production Science, 59(3):479-485.

Monteiro, C. C. F., Melo, A. A. S., Ferreira, M. A., Campos, J. M. S., Souza, J. S. R., Silva, E. T. S., . . Silva, E. C. (2014). Replacement of wheat bran with spineless cactus (Opuntia ficus indica Mill cv Gigante) and urea in the diets of Holstein x Gyr heifers. Tropical Animal Health and Production, 46(7):1149-1154.

Oliveira, J. P. F., Ferreira, M. A., Alves, A. M. S. V., Melo, A. C. C., Andrade, I. B., Suassuna, J. M. A., . . Silva, J. L. (2017). Spineless cactus as a replacement for sugarcane in the diets of finishing lambs. Tropical Animal Health and Production, 49(1):139-144.

Oliveira, J. P. F., Ferreira, M. A., Alves, A. M. S. V., Melo, A. C. C., Andrade, I. B., Urbano, S. A., . . . Barros Melo, T. T. (2018). Carcass characteristics of lambs fed spineless cactus as a replacement for sugarcane. Asian-Australasian Journal of Animal Sciences, 31(4):529-536.

Pessoa, R. A. S., Ferreira, M. A., Silva, F. M., Bispo, S. V., Wanderley, W. L. \& Vasconcelos, P. C. (2013). Diferentes suplementos associados à palma forrageira em dietas para ovinos: consumo, digestibilidade aparente e parâmetros ruminais. Revista Brasileira de Saúde e Produção Animal, 14(3):508-517.

Ramos, A. O., Ferreira, M. A., Véras, A. S. C., Costa, S. B. M., Conceição, M. G., Silva, E. C., Souza, A. R. D. L. (2013). Diferentes fontes de fibra em dietas a base de palma forrageira na alimentação de ovinos. Revista Brasileira de Saúde e Produção Animal, 14(4):648-659.

Rekik, M., Ben Salem, H., Lassoued, N., Chalouati, H. \& Ben Salem, I. (2010). Supplementation of Barbarine ewes with spineless cactus (Opuntia ficus-indica f. inermis) cladodes during late gestationearly suckling: Effects on mammary secretions, blood metabolites, lamb growth and postpartum ovarian activity. Small Ruminant Research, 90(1-3):53-57.

Rocha Filho, R. R. (2012). Palma gigante e genótipos resistentes à cochonilha do carmim em dietas para ruminantes. Univrsidade Federal Rural de Pernambuco, Recife, Pernambuco, Brasil. 
Santos, A. O. A., Batista, A. M., Mustafa, A., Amorim, G. L., Guim, A., Moraes, A. C., . . Andrade, R. (2010). Effects of Bermudagrass hay and soybean hulls inclusion on performance of sheep fed cactus-based diets. Tropical Animal Health and Production, 42(3):487-494.

Santos, D., Farias, I., Lira, M., Tavares Filho, J., Santos, M. \& Arruda, G. (1997). A palma forrageira (Opuntia ficus-indica Mill e Nopalea cochenillifera Salm-Dyck) em Pernambuco: cultivo e utilização. Recife: IPA.

Santos, D. C., Silva, M. C., Dubeux Júnior, J. C. B., Lira, M. A. \& Silva, R. M. (2013). Estratégias para uso de cactáceas em zonas semiáridas: novas cultivares e uso sustentável das espécies nativas. Revista Científica de Produção Animal, 15(2):111-121.

Santos, R. D., Neves, A. L. A., Santos, D. C., Pereira, L. G. R., Gonçalves, L. C., Ferreira, A. L., . . . Sollenberger, L. E. (2018). Divergence in nutrient concentration, in vitro degradation and gas production potential of spineless cactus genotypes selected for insect resistance. The Journal of Agricultural Science, 156(3):450-456.

Silva, C. C. F. \& Santos, L. C. (2006). Palma forrageira (Opuntia fícus-indica Mill) como alternativa na alimentação de ruminantes. Revista Eletrônica de Veterinária, 7(10):1-13.

Silva, E. T. S., Melo, A. A. S., Ferreira, M. d. A., Oliveira, J. C. V. d., Santos, D. C. d., Silva, R. C. \& Inácio, J. G. (2017). Acceptability by Girolando heifers and nutritional value of erect prickly pear stored for different periods. Pesquisa Agropecuária Brasileira, 52(9):761-767.

Silva, L. M., Fagundes, J. L., Viegas, P. A. A., Moreira, A. L. \& Backes, A. A. (2014). Produtividade da palma forrageira cultivada em diferentes densidades de plantio. Ciência Rural, 44(11):2064-2071.

Silva, R. C., Ferreira, M. A., Oliveira, J. C. V., Santos, D. C., Gama, M. A. S., Chagas, J. C. C., .. . Pereira, L. G. R. (2018). Orelha de Elefante Mexicana (Opuntia stricta [Haw.] Haw.) spineless cactus as an option in crossbred dairy cattle diet. South African Journal of Animal Science, 48(3):516-525.

Silva, R. R. \& Sampaio, E. V. S. B. (2015). Palmas forrageiras Opuntia fícus-indica e Nopalea cochenillifera: sistemas de produção e usos. Revista Geama, 1(2):151-161.

Siqueira, M. C. B., Ferreira, M. A., Monnerat, J. P. I. S., Silva, J. L., Costa, C. T. F., Conceição, M. G., . . . Melo, T. T. B. (2017). Optimizing the use of spineless cactus in the diets of cattle: Total and partial digestibility, fiber dynamics and ruminal parameters. Animal Feed Science and Technology, 22656-64.

Siqueira, M. C. B., Ferreira, M. A., Monnerat, J. P. I. S., Silva, J. L., Costa, C. T. F., Conceição, M. G., Chagas, J. C. C. (2018). Nutritional performance and metabolic characteristics of cattle fed spineless cactus. Journal of Agricultural Science and Technology, 20(1):13-22.

Souza, A. E. F., Nascimento, L. C., Araújo, E., Lopes, E. B. \& Souto, F. M. (2010). Ocorrência e identificação dos agentes etiológicos de doenças em palma forrageira (Opuntia ficus-indica Mill.) no semiárido paraibano. Biotemas, 23(3):11-20.

Souza, E. J., Guim, A., Batista, A. M. V., Santos, K. L., Silva, J. R., Morais, N. A. P. \& Mustafa, A. F. (2009). Effects of soybean hulls inclusion on intake, total tract nutrient utilization and ruminal fermentation of goats fed spineless cactus (Opuntia ficus-indica Mill) based diets. Small Ruminant Research, 85(1):63-69.

Vasconcelos, A. G. V., Lira, M. A., Cavalcanti, V., Santos, M. V. F. \& Willadino, L. (2009). Seleção de clones de palma forrageira resistentes à cochonilha-do-carmim (Dactylopius sp). Revista Brasileira de Zootecnia, 38(5):827-831.

Vieira, E. L., Batista, A. M. V., Guim, A., Carvalho, F. F. R., Nascimento, A. C., Araújo, R. F. S. \& Mustafa, A. F. (2008). Effects of hay inclusion on intake, in vivo nutrient utilization and ruminal fermentation of goats fed spineless cactus (Opuntia ficus-indica Mill) based diets. Animal Feed Science and Technology, 141((3-4)):199-208.

Weimer, P. J. (1998). Manipulating ruminal fermentation: a microbial ecological perspective. Journal of Animal Science, 76(12):3114-3122.

Recebido: 18 de janeiro, 2019.

Aprovado: 18 de fevereiro, 2019

Publicado: 6 de março, 2019

Licenciamento: Este artigo é publicado na modalidade Acesso Aberto sob a licença Creative Commons Atribuição 4.0 (CC-BY 4.0), a qual permite uso irrestrito, distribuição, reprodução em qualquer meio, desde que o autor e a fonte sejam devidamente creditados. 